\title{
PERINATAL COMPUTING IN 1987: AN OVERVIEW
}

\author{
Robert J. Sokol, M. D. \\ Ivan E. Zador, Ph. D. \\ Department of Obstetrics/Gynecology \\ Wayne State University/Hutzel Hospital, Detroit USA
}

This supplemental issue of the Journal of Perinatal Medicine consists of short papers and abstracts accepted for presentation at the First World Symposium on Computers in the Care of the Mother. Fetus, and Newborn, held in Vienna, Austria, from 8 March 1987 through 12 March 1987. The response to the call for papers was overwhelming, necessitating a late and previously unplanned extension of the meeting by a full day to make it possible to present as much of the excellent work that was submitted as possible. The papers submitted were refereed by the Scientific Review Committee of the Symposium. Of those submitted, a total of 250 abstracts were accepted for presentation and publication. These papers came from 30 countries. The manuscripts are included in this Supplement, in their planned order of presentation at the Symposium.

Once a decision had been reached concerning the papers to be accepted, the Scientific Review Committee was faced with the difficult task of deciding on an appropriate forum for presentation of each. It is, of course, always difficult to judge the quality of work from relatively short communications and, indeed, the committee believes that there is little, if any, difference in quality of the work to be presented in the General Sessions, compared with the Split Sessions, Poster Sessions and Demonstrations. As a matter of fact, several authors requested that their work be presented as a Poster or a Demonstration, based on the anticipated size of the group of individuals interested, potential language barriers and the type of material to be presented. Important in the decision for presentation in the General Sessions were criteria which included potential broad interest by many in attendance at the Symposium and broad representation, both in terms of geographic balance and of the full range of research activities going on within this discipline.

Individuals reading this Supplement and/or attending the Symposium will undoubtedly draw their own conclusions regarding general trends in perinatal computing over the last ten to fifteen years and the current status of the field. Based, however, upon initial review of the manuscripts by the scientific review committee, some preliminary observations are warranted.

Large perinatal databases continue under active development in many countries. Typically, the goal of developing and using such databases is improved public health with improved quality of perinatal care. A number of new applications are presented. Smaller perinatal databases, ie, those covering smaller regions or specific hospital services, are broadly represented in the following manuscripts. Whereas 5 to 10 years ago, such databases were relatively rare, the advent of inexpensive hardware and sophisticated database management systems for micro computers apparently has produced a rapid dispersion of this type of approach to perinatal record keeping, as well as clinical report generation and support of quality assurance at the regional and local levels.

There appears to have been a resurgence of interest in computer-based electronic fetal monitoring. Again, the impetus appears to be the availability of powerful microcomputers. Such applications were previously limited to minis and mainframes. Work continues in the area of computer-based pattern recognition and evaluation of beat-to-beat variability. There appears to be increased recognition of the problems of storage of the massive amounts of data generated during fetal monitoring. Further, there appears to be increased emphasis on assessment of the fetus during the antepartum period with particular attention 
to fetal activity periods.

A new method of fetal assessment has assumed major importance. This area of activity involves computer-based assessment of fetal vascular flow, particularly as used for the diagnosis and management of the growth retarded fetus. There are also a number of other computer-based ultrasound systems being reported. Computerization of fetal mensuration now appears to be mainly in the area of report generation, since much of the developmental work has apparently been completed.

With regard to neonatal applications, it appears that most of the work involves bedside (incubator side) computing. Much of this work is very practical, with emphasis on calculation of nutritional requirements and generation of discharge summaries and discharge letters.

Some of the special applications are perhaps best termed "off-beat". They are certainly fascinating. There appears to be a new emphasis on computer-based telemetry of data obtained in the home environment, with assessment of potential cost savings from such applications. In addition, several new computer-aided education systems have been developed. Based on work in this area, one might anticipate much broader application of such technology in the near future.

The big news in perinatal computing is expert systems. Such emphasis undoubtedly grows in part from the information explosion being experienced in perinatology. Some of these expert systems use standard procedure oriented programming techniques, with complex decision trees. Some of these applications can now be used at the bedside. Even more exciting, however, are the knowledge engineering rule base systems, typically termed artificial intelligence systems. Perinatal investigators are not awaiting the fifth generation computers being developed in Japan. They are moving ahead rapidly, using micro computer based Al languages such as LISP and PROLOG.

In conclusion, with each successive meeting, the area of perinatal computing increases in breadth, depth and excitement. The Journal of Perinatal Medicine, by publication of the short papers in this Supplement and of up to 15 full manuscripts in a later issue, will help move research and development in this field along an accelerated pace. We wish to thank Professor Dudenhausen, the Editor-in-Chief of the Journal, Mrs. Mannaa from de Gruyter Publisher and the Milupa Company which provided financial support for this publication. 